\title{
Hipertensão Arterial Sistémica Secundária: Incertezas do Diagnóstico
}

\author{
Secondary Arterial Hypertension: Uncertainties in \\ Diagnosis
}

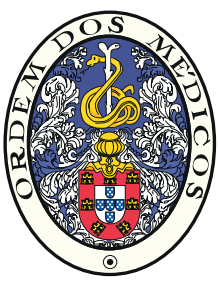

Paulo Gomes DINIS $\mathbb{1}^{1}$, Maria Carmo CACHULO ${ }^{1}$, Andreia FERNANDES ${ }^{1}$, Luís PAIVA ${ }^{1}$, Lino GONÇALVES ${ }^{1}$ Acta Med Port 2017 Jun;30(6):493-496 - https://doi.org/10.20344/amp.8007

\begin{abstract}
RESUMO
A hipertensão arterial sistémica configura-se hoje como um problema de saúde pública mundial, sendo a taxa de prevalência em Portugal de 26,9\%. De acordo com a sua etiologia classifica-se em hipertensão arterial sistémica primária ou secundária. Em cerca de $90 \%$ dos casos, não é possível estabelecer uma causa e por isso a hipertensão arterial sistémica denomina-se primária. Nos restantes $5 \%$ a $10 \%$, pode ser identificada uma causa secundária, potencialmente tratável. Para que o estudo da hipertensão arterial sistémica secundária seja custo-eficaz, torna-se essencial perceber quais os doentes a investigar, com que meios, e qual a melhor estratégia a adotar. As principais causas apontadas como responsáveis pela hipertensão arterial sistémica secundária são: patologia renal; endócrina; vascular e síndrome da apneia obstrutiva do sono. Entre estas, algumas são consensuais e outras mais controversas na literatura. A este propósito apresentamos dois casos de hipertensão arterial sistémica, cuja etiologia potencialmente secundária ainda é foco de debate.
\end{abstract}

Palavras-chave: Artéria Renal/anomalias congénitas; Doenças Renais Quísticas; Hipertensão/diagnóstico; Pressão Sanguínea

\section{ABSTRACT}

Arterial hypertension is regarded today as a global public health problem, and the prevalence rate in Portugal is $26.9 \%$. According to the etiology, is classified into primary or secondary arterial hypertension. In about $90 \%$ of cases it is not possible to establish a cause, so is called primary arterial hypertension. In the remaining 5 to $10 \%$, it can be identified secondary causes, which are potentially treatable. For secondary arterial hypertension study to be cost-effective, it is essential to understand which patients investigate, and evaluate the best strategy to adopt. The main causes identified as responsible for secondary arterial hypertension are: kidney disease; endocrine and vascular diseases and obstructive sleep apnea. Among these some are consensual, and others more controversial in the literature. In this regard we present two cases of arterial hypertension, which are potentially secondary in etiology, but still focus of debate.

Keywords: Blood Pressure; Hypertension/diagnosis; Kidney Diseases, Cystic; Renal Artery/abnormalitie

\section{INTRODUÇÃO}

A hipertensão arterial (HTA) é um fator de risco major para doença cardiovascular. ${ }^{1,2}$ A HTA secundária define-se como um aumento da pressão arterial devido a uma causa identificável, ${ }^{3}$ ocorrendo em $5 \%-10 \%$ de todos os casos de HTA. ${ }^{4}$ Devido a esta baixa percentagem, é primordial saber que doentes devemos estudar. A história clínica e o exame objetivo são instrumentos valiosos para guiar o clínico nesta seleção.

A HTA secundária está associada a uma patologia potencialmente tratável, requerendo alto grau de suspeita. $\mathrm{Na}$ pesquisa inicial devemos excluir a HTA de bata branca, aferir a adesão terapêutica e identificar sinais e sintomas indicadores de HTA secundária. Devemos estudar jovens sem outros fatores de risco cardiovasculares (FRCV); HTA de início recente ou previamente controlada; HTA com lesão de órgão alvo e HTA resistente. ${ }^{3}$ Esta última define-se como persistência de valores aumentados, apesar da instituição de medidas gerais e administração em dose ótima de três ou mais antihipertensivos, sendo um deles diurético. ${ }^{5,6}$

As causas HTA secundária são múltiplas. Nos jovens a patologia do parênquima renal e as doenças vasculares são comuns. ${ }^{3}$ Nos adultos a síndrome da apneia obstrutiva do sono é a causa mais prevalente. ${ }^{3} \mathrm{~A}$ tabela 1 refere alguns distúrbios responsáveis por HTA secundária, sugerin- do exames de rastreio e de confirmação diagnóstica. Esta orientação não existe para os casos mais raros. Nestes, a abordagem tem de ser multidisciplinar e individualizada.

Mesmo com tratamento apropriado, nem sempre se alcança o estado normotenso, dado que muitos doentes apresentam concomitantemente hipertensão essencial. ${ }^{4} \mathrm{~A}$ maioria dos jovens responde bem a um tratamento dirigido, contrariamente aos mais velhos. ${ }^{3}$

Reportamos dois casos de HTA, cuja etiologia permanece controversa.

\section{CASO 1}

Indivíduo do género feminino, 48 anos, com antecedentes pessoais de HTA há mais de uma década, dislipidémia, diabetes mellitus tipo II, doença renal crónica por nefropatia diabética diagnosticada há cinco anos atualmente em estádio II. Sem outros FRCV. O exame objetivo não apresentava alterações. Medicada desde há seis meses com irbesartan e hidroclorotiazida 300/12,5 mg um comprimido/ dia (id), furosemida $40 \mathrm{mg}$ id, lercanidipina $10 \mathrm{mg}$ id e rilmenidina $1 \mathrm{mg}$ id. A monitorização ambulatória da pressão arterial (MAPA) das 24 horas revelou uma HTA não controlada, com predomínio noturno e perfil non-dipper.

Durante a investigação a ecografia com Doppler renal

1. Serviço de Cardiologia. Centro Hospitalar e Universitário de Coimbra. Coimbra. Portugal.

$\triangle$ Autor correspondente: Paulo Gomes Dinis. paulogdinis@gmail.com

Recebido: 04 de julho de 2016 - Aceite: 24 de janeiro de 2017 | Copyright ( ) Ordem dos Médicos 2017 
Tabela 1 - Exames de rastreio relativos a cada causa específica de HTA secundária e a sugestão da confirmação diagnóstica ${ }^{2}$

\begin{tabular}{|c|c|c|c|}
\hline Causa secundária & Prevalência & Screening & Diagnóstico \\
\hline $\begin{array}{l}\text { Síndrome da apneia } \\
\text { obstrutiva do sono }\end{array}$ & $>5 \%-15 \%$ & $\begin{array}{l}\text { Questionário de screening } \\
\text { (ex: Epworth Sleepiness scale) }\end{array}$ & Polissonografia \\
\hline HTA renoparenquimatosa & $1,6 \%-8 \%$ & Creatinina, ecografia renal & Biópsia renal \\
\hline HTA renovascular & $1 \%-8 \%$ & Ecografia renal & $\begin{array}{l}\text { Eco Doppler renal; Angio-TC } \\
\text { abdominal; Angiografia renal }\end{array}$ \\
\hline Hiperaldostronismo $1^{\circ}$ & $1,4 \%-10 \%$ & Hipocaliémia, razão aldosterona e renina & $\begin{array}{l}\text { TC abdominal; RM abdominal; } \\
\text { Punção venosa da supra-renal }\end{array}$ \\
\hline Patologia tiroideia & $1 \%-2 \%$ & TSH e T4 L & Ecografia da tiróide \\
\hline Síndrome de Cushing & $0,5 \%$ & Cortisol - urina de 24 horas & $\begin{array}{l}\text { ACTH plasmático; Teste de supressão } \\
\text { com dexametasona; TC ou RM das } \\
\text { supra renais }\end{array}$ \\
\hline Feocromocitoma & $0,2 \%-0,5 \%$ & $\begin{array}{l}\text { Metanefrinas plasmáticas, catecolaminas } \\
\text { - urina } 24 \text { horas }\end{array}$ & $\begin{array}{l}\text { TC ou RM abdominal; Cintigrafia com } \\
\text { MIBG. }\end{array}$ \\
\hline Coartação da aorta & $<1 \%$ & Ecocardiograma & Aortografia \\
\hline
\end{tabular}

TC: Tomografia computorizada; RM: Ressonância magnética; TSH: Hormona estimulante da tiróide; T4L: Tiroxina livre; ACTH: Hormona adrenocorticotrófica; MIGB: Metaiodobenzilguanidina.

mostrou o rim esquerdo com diminuição da diferenciação parênquimo-sinusal. Por deficiente visualização das artérias renais, realizou angio-tomografia computorizada (TC) renal, que documentou a presença de duas artérias renais acessórias à direita, e a duplicação da artéria renal esquerda com uma artéria acessória no polo superior (Fig.1).

\section{Comentário}

A existência de artérias renais acessórias ocorre em aproximadamente $26 \%$ dos indivíduos. ${ }^{7}$ A relação causal com a HTA é controversa.

Alguns autores defendem que as artérias renais acessórias podem causar HTA, dado serem mais longas e com menor calibre, aumentando a resistência e predispondo

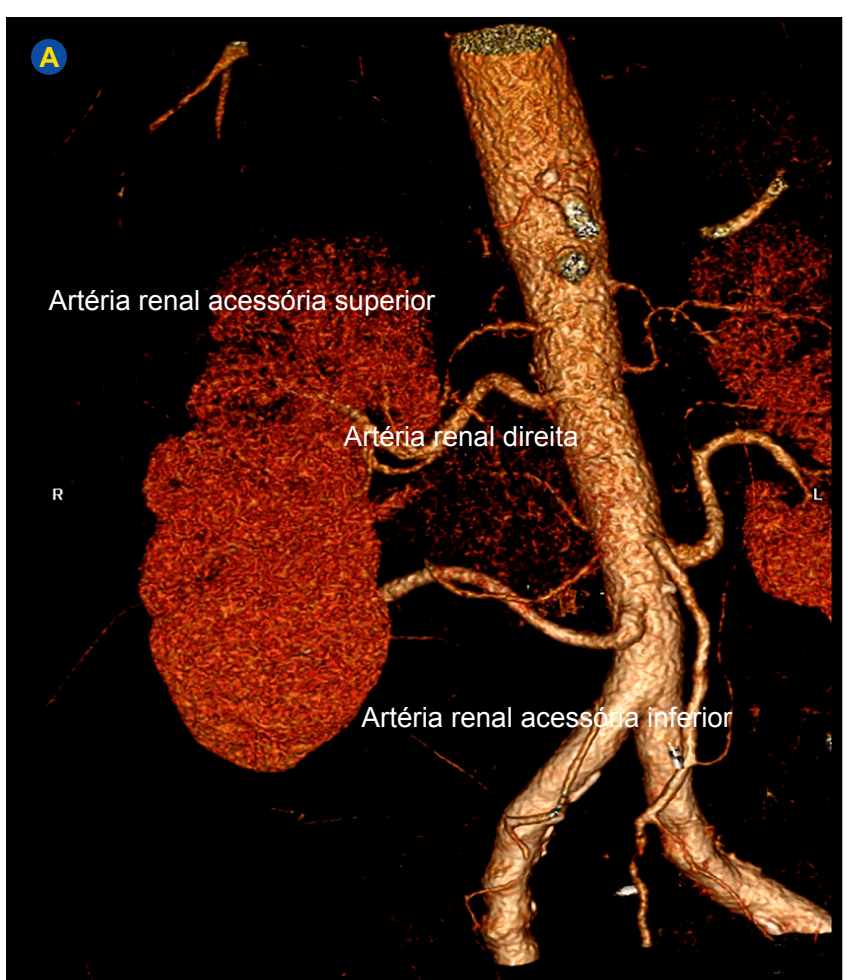

a alterações hemodinâmicas que levam à hipoperfusão e disfunção renal. ${ }^{8}$ Desta hipoperfusão resulta um estado de hiperreninémia contribuindo para a HTA pela ativação do sistema-renina angiotensina-aldosterona (SRAA). Está documentada na literatura, a normalização da pressão arterial após ressecção das artérias renais acessórias. ${ }^{9}$

Outros autores ${ }^{10}$ afirmam que as artérias renais acessórias não aumentam o risco de HTA, referindo que a prevalência de estenose da artéria renal nos doentes com e sem artérias renais acessórias, não é estatisticamente significativa.

Neste caso, de doente hipertensa, diabética e com doença renal crónica torna-se dificil quantificar o contributo das artérias renais acessórias para a origem ou

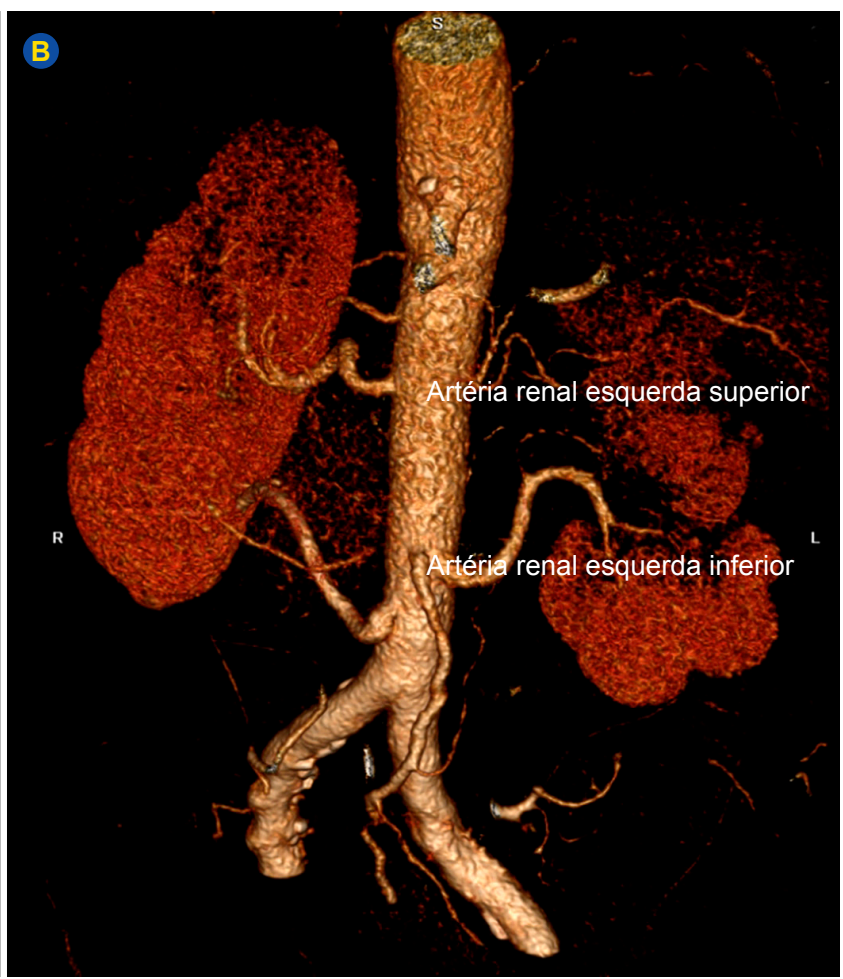

Figura 1 - Artéria renal direita acompanhada da artéria renal acessória superior e inferior (A). Duplicação da artéria renal esquerda (B). 
agravamento da HTA. Mantivemos a doente apenas com terapêutica antihipertensora, ajustando-a para as doses máximas toleradas (irbesartan com hidroclorotiazida para 300/25 mg id e lercanidipina para $20 \mathrm{mg}$ id), conseguindo-se um perfil tensional com valores sistólicos $<140 \mathrm{mmHg}$ e diastólicos $<90 \mathrm{mmHg}$. Por estes valores não serem os pretendidos numa doente diabética, ponderou-se a realização de uma angiografia renal para diagnóstico de estenose ou displasia fibromuscular. Outra opção de tratamento seria a desnervação renal. ${ }^{11}$ Após discussão com a doente, explicando os riscos/benefícios, optou-se por manutenção do tratamento médico otimizado.

\section{CASO 2}

Indivíduo do género masculino, 58 anos, com hipercolesterolémia e HTA diagnosticada hà oito anos, previamente controlado, mas com valores elevados desde há um ano. Sem outros FRCV. Sem alterações ao exame objetivo e sem lesões de orgão alvo. Medicado com losartan e hidroclorotiazida 100/12,5 mg id. Por queixas de desconforto lombar aquando da micção, realizou ecografia renal que identificou o rim esquerdo de dimensões aumentadas, à custa de cinco formações quísticas (Fig. 2). Pediu-se TC renal que caracterizou estes quistos, o maior com $9,2 \mathrm{~cm}$, condicionando proeminência do sistema piélico (Fig. 3).

Procedeu-se à citologia aspirativa, cujo resultado levantou suspeitas de malignidade. Realizou-se nefrourectomia esquerda por laparoscopia, cujas biópsias foram negativas para a existência de doença neoplásica.

\section{Comentário}

A associação de quistos renais e HTA é controversa. A prevalência dos quistos renais aumenta com a idade, assim como a incidência de $\mathrm{HTA}^{5}$. Fatores confundidores como a idade, género, taxa de filtração glomerular (TFG) e proteinúria devem ser examinados. ${ }^{12}$

Alguns autores afirmam que os quistos renais são a causa da HTA. Referem que a expansão dos quistos e a

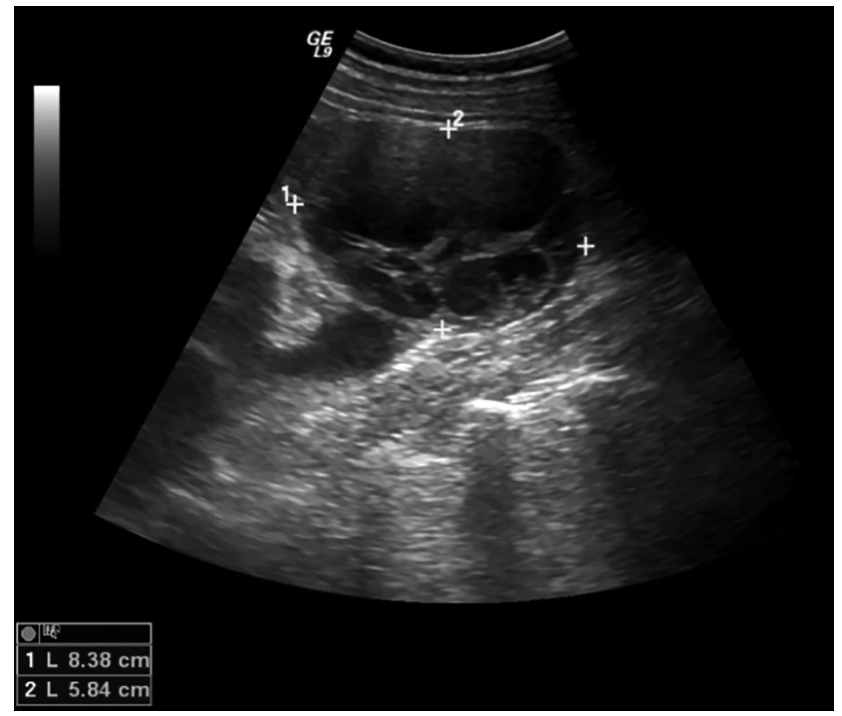

Figura 2 - Ecografia renal: rim esquerdo com múltiplas formações quísticas

compressão arterial ativam o SRAA provocando isquemia renal. ${ }^{13} \mathrm{~A}$ probabilidade de um quisto renal acionar o SRAA por compressão arterial está dependente da localização, tamanho e número. ${ }^{12}$ Outra explicação sugere que a perda de nefrónios associada à idade, está envolvida tanto no desenvolvimento de HTA, como na formação de quistos renais. ${ }^{14} \mathrm{~A}$ perda de nefrónios leva a uma hipertrofia e hiperplasia das células tubulares, podendo originar quistos. ${ }^{5}$ Relativamente ao tratamento, existe uma aparente associação entre a drenagem ou remoção dos quistos e a redução da pressão arterial no follow-up. ${ }^{13,15}$

Neste doente, verificou-se uma melhoria progressiva do perfil tensional, documentado com MAPA, tendo sido possível reduzir a medicação anti-hipertensora, estando atualmente bem controlado com losartan $50 \mathrm{mg}$ id, apontando os quistos renais como a provavél causa de HTA secundária sobreposta numa HTA primária residual.
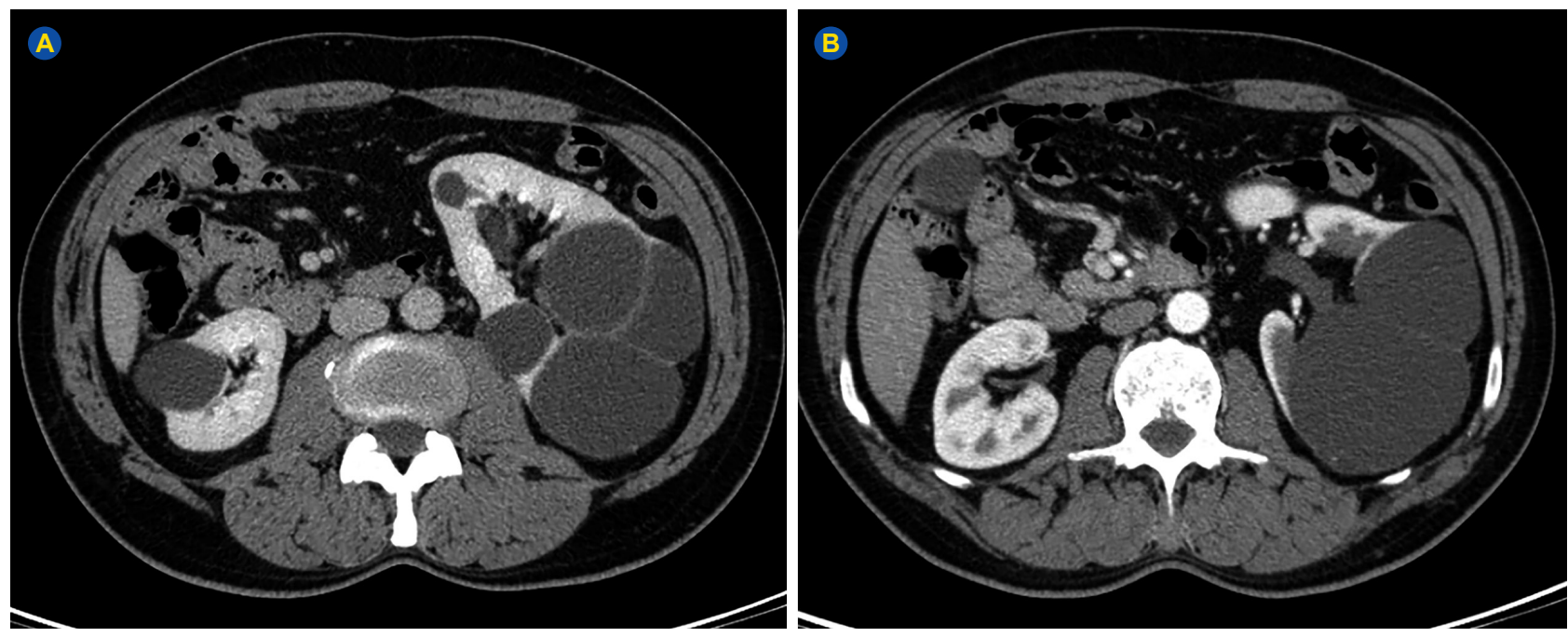

Figura 3 - Tomografia renal: múltiplos quistos no rim esquerdo na vertente posterior do parênquima, condicionando proeminência do sistema piélico (A). Quisto renal simples (B). 


\section{CONCLUSÕES}

O estudo da HTA secundária de causas raras é complexo. Os casos ilustram alterações importantes da vasculatura e do parênquima renal. No primeiro, manteve-se a incerteza do contributo das artérias renais acessórias para a HTA. No segundo, a evolução clínica permitiu-nos apontar como provável a etiologia secundária, contudo a bibliografia não é consensual.

\section{REFERÊNCIAS}

1. Macedo ME, Ferreira RC. Programa Nacional para as doenças cerebro e cardiovasculares. Lisboa: Direção Geral da Saúde; 2013.

2. Chobanian AV, Bakris GL, Black HR, Cushman WC, Green LA, Izzo JL $\mathrm{Jr}$, et al. Seventh report of the joint national committee on prevention, detection, evaluation, and treatment of high blood pressure: the JNC 7 report. JAMA. 2003;290:2560-72.

3. Rimoldi SF, Scherrer U; Messerli FH. Secondary arterial hypertension: when, who, and how to screen? Eur Heart J. 2014;35:1245-54.

4. Chiong JR, Aronow WS, Khan IA, Nair CK, Vijayaraghavan K, Dart RA, et al. Secondary hypertension: Current diagnosis and treatment. Int $\mathrm{J}$ Cardiol. 2007;124:6-21.

5. Mancia G, De Backer G, Dominiczak A, Cifkova R, Fagard R, Germano $G$, et al. Guidelines for the management of arterial hypertension: the task force for the management of arterial hypertension of the European Society of Hypertension (ESH) and of the European Society of Cardiology (ESC). Blood Press. 2007;16:135-232.

6. Calhoun DA, Jones D, Textor S, Goff DC, Murphy TP, Toto RD, et al. Resistant hypertension: diagnosis, evaluation, and treatment: a scientific statement from the American Heart Association Professional Education Committee of the Council for High Blood Pressure Research. Circulation. 2008;117:e510-26.

7. Lippert H, Pabst R. Arterial variations in man. Classifications and frequency. Munich: Bergmann Publishers; 1985.

\section{CONFLITOS DE INTERESSE}

Os autores declaram não terem qualquer conflito de interesse relativamente ao presente artigo.

\section{FONTES DE FINANCIAMENTO}

Os autores declaram não ter recebido subsídios ou bolsas para a elaboração do artigo.

8. Glodny B, Cromme S, Reimer P, Lennarz M, Winde G, Vetter $H$. Hypertension associated with multiple renal arteries may be renindependent. J Hypertension. 2000;18:1437-44.

9. Kem DC, Lyons DF, Wenzl J, Halverstadt D, Yu X. Renin-dependent hypertension caused by nonfocal stenotic aberrant renal arteries: proof of a new syndrome. Hypertension. 2005;46:380-5.

10. Gupta A, Tello R. Accessory renal arteries are not related to hypertension risk: a review of MR angiography data. AJR Am J Roentgenol. 2004;182:1521-4

11. Silva J, Costa M, Gersh BJ, Gonçalves L. Renal denervation in the era of HTN-3. Comprehensive review and glimpse into the future. J Am Soc Hypertens. 2016;10:656-70.

12. Chin HJ, Ro H, Lee HJ, Na KY, Chae DW. The clinical significances of simple renal cyst: is it related to hypertension or renal dysfunction? Kidney Int. 2006;70:1468-73.

13. Pedersen JF, Emamian SA, Nielsen MB. Significant association between simple renal cysts and arterial blood pressure. Br J Urol. 1997;79:68891.

14. Keller G, Zimmer G, Mall G, Ritz E, Amann K. Nephron number in patients with primary hypertension. N Engl J Med. 2003;348:101-8.

15. Grantham JJ. Acquired cystic kidney disease. Kidney Int. 1991;40:14352.
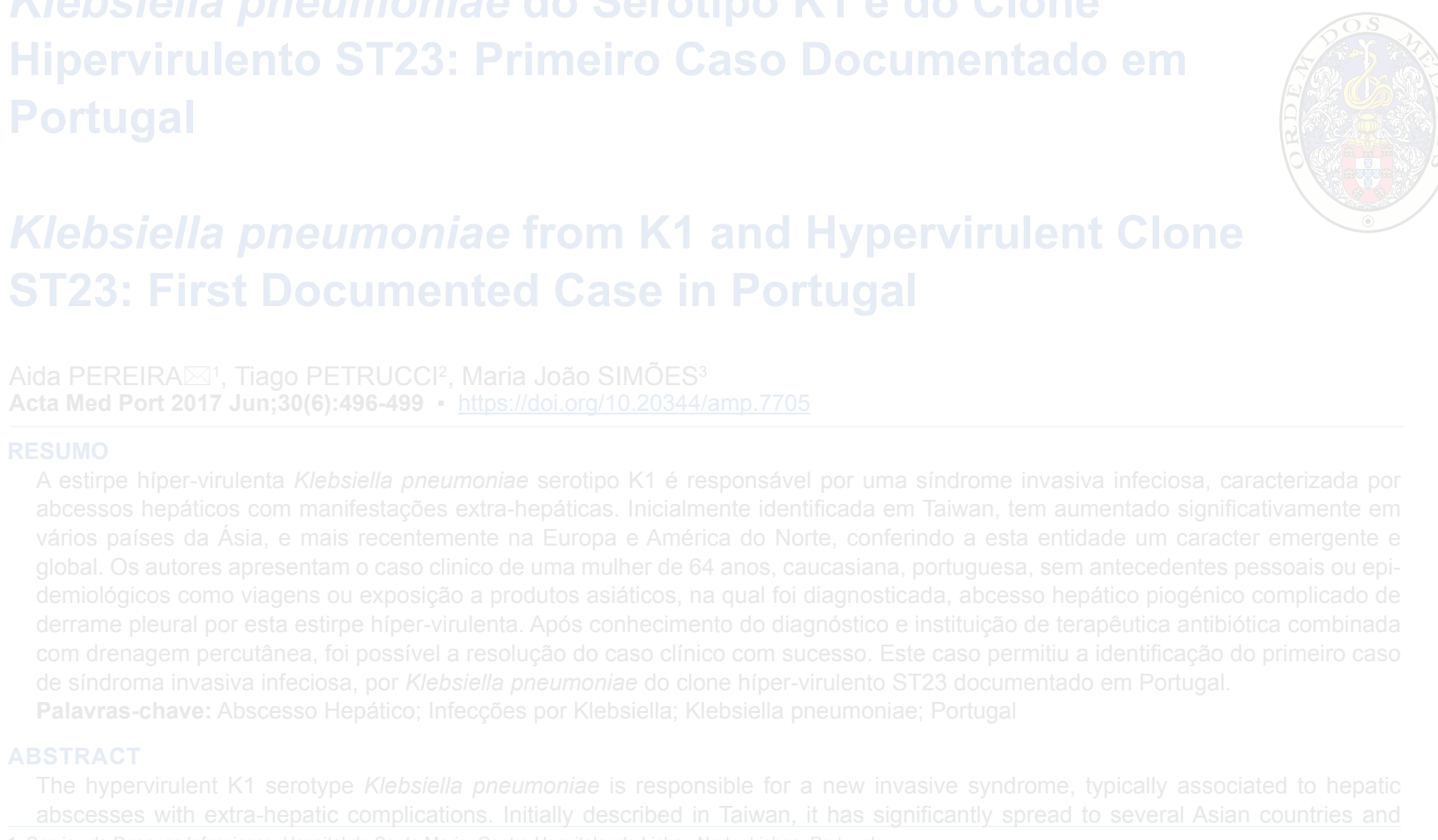